\title{
Costs associated with tuberculosis diagnosis and treatment in Yemen for patients and public health
} services

G.Q. Othman, ${ }^{\top}$ M.I.M. Ibrahim ${ }^{2}$ and Y.A. Raja' ${ }^{3}$

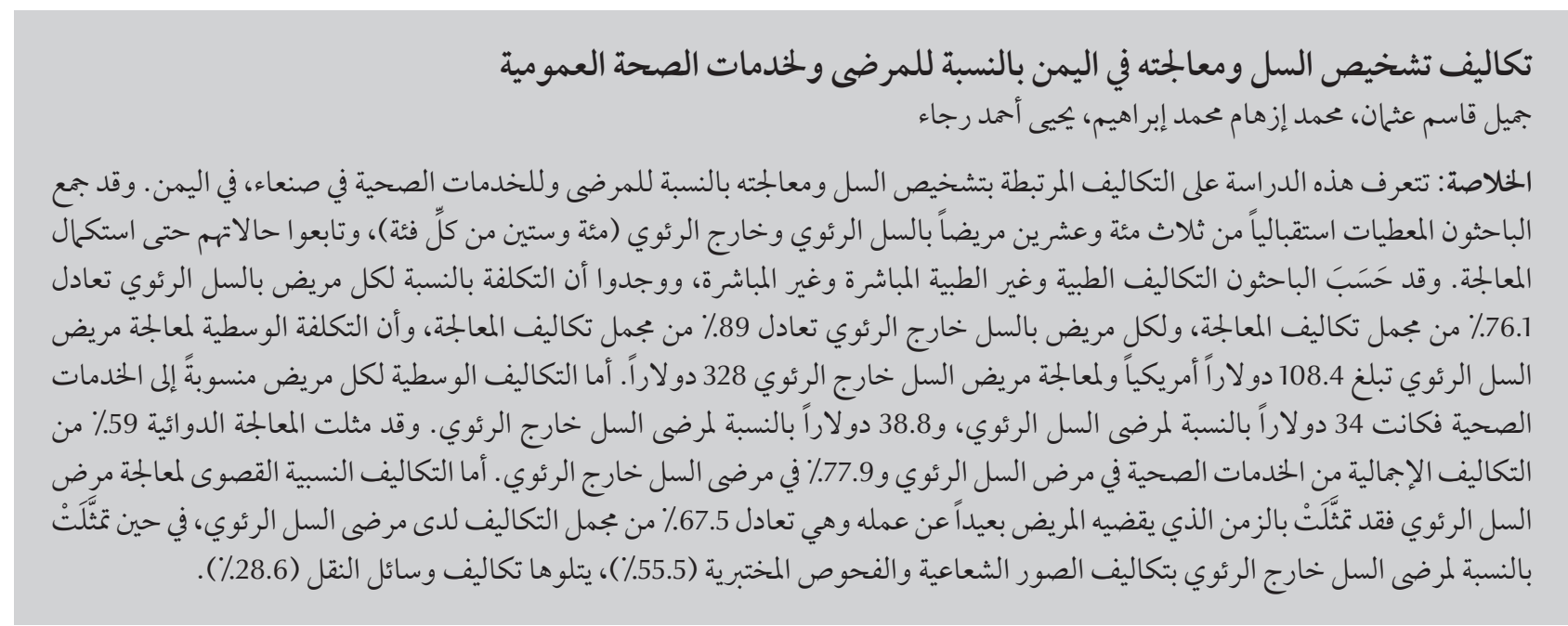

ABSTRACT This study determined the costs associated with tuberculosis (TB) diagnosis and treatment for the public health services and patients in Sana'a, Yemen. Data were collected prospectively from 320 pulmonary and extrapulmonary TB patients (160 each) who were followed until completion of treatment. Direct medical and nonmedical costs and indirect costs were calculated. The proportionate cost to the patients for pulmonary TB and extrapulmonary TB was $76.1 \%$ and $89.4 \%$ respectively of the total for treatment. The mean cost to patients for pulmonary and extrapulmonary TB treatment was US\$108.4 and US\$328.0 respectively. The mean cost per patient to the health services for pulmonary and extrapulmonary TB treatment was US\$34.0 and US\$ 38.8 respectively. For pulmonary and extrapulmonary TB, drug treatment represented $59.3 \%$ and $77.9 \%$ respectively of the total cost to the health services. The greatest proportionate cost to patients for pulmonary TB treatment was time away from work $(67.5 \%$ of the total cost), and for extrapulmonary TB was laboratory and X-ray costs (55.5\%) followed by transportation (28.6\%).

Coûts associés au diagnostic et au traitement de la tuberculose pour les patients et les services de santé publics au Yémen RÉSUMÉ La présente étude a déterminé les coûts associés au diagnostic et au traitement de la tuberculose pour les services de santé publics et pour les patients à Sanaa (Yémen). Les données ont été recueillies de façon prospective auprès de 320 patients atteints de tuberculose pulmonaire et extrapulmonaire (160 pour chaque forme), suivis jusqu'à la fin de leur traitement. Les coûts médicaux et non médicaux directs et les coûts indirects ont été calculés. La part du coût supportée par les patients était de 76,1 \% du coût total du traitement dans le cas de la tuberculose pulmonaire, et de 89,4 \% dans le cas de la tuberculose extrapulmonaire. Le coût moyen du traitement de la tuberculose pulmonaire et extrapulmonaire pour les patients était respectivement de 108,4 USD et 328,0 USD, ce qui équivalait à un coût moyen par patient de 34,0 USD et 38,8 USD, respectivement. Pour les services de santé, le traitement médicamenteux de la tuberculose pulmonaire et extrapulmonaire représentait $59,3 \%$ et $77,9 \%$ respectivement du coût total. Pour les patients atteints de tuberculose pulmonaire, l'absence du travail représentait le coût le plus important du traitement $(67,5 \%$ du coût total) ; pour les patients atteints de tuberculose extrapulmonaire, les dépenses liées aux analyses de laboratoire et auxradiographies (55,5\%) étaient les coûts proportionnellement les plus importants, suivies par les frais de transport $(28,6 \%)$.

${ }^{7}$ Social \& Administrative Pharmacy Discipline, School of Pharmaceutical Sciences, Universiti Sains Malaysia, Penang, Malaysia (Correspondence to G.Q. Othman: gamilqasem@yahoo.com). 2Pharmacy Practice Department, College of Pharmacy, Qassim University, Buraidah, Al Qassim, Saudi Arabia. ${ }^{3}$ Faculty of Medicine and Health Sciences, Sana'a University, Sana'a, Yemen.

Received: 16/08/10; accepted: 28/10/10 


\section{Introduction}

Tuberculosis (TB) infects one-third of the world's population [1]. It is ranked seventh as cause of death worldwide $[2,3]$ and is one of the common causes of death among young adults [4]. Thus the effect of TB on the society is massive.

From the economic perspective, about $75 \%$ of TB cases are in the productive age group (15-54 years), and $95 \%$ of these patients and $99 \%$ of the deaths from TB occur in developing countries [5]. The estimated cost of TB treatment in developed countries ranges from US\$ 276 to US\$ 1546 per person for the entire treatment, while for multi-drug resistant $\mathrm{TB}$ it ranges from US\$ 1000 to US\$ 10000 [6]. The cost of the effective TB drug is considered the most important component of the actual expenditure for treating TB. However, other direct and indirect costs for TB treatment must also be calculated. A study in Malaysia found the total cost for the TB patient who completed their treatment successfully was US\$ 916.40 [7] while in India the total cost was US\$ 7.5 [8]. In the Netherlands a study compared the direct and indirect costs of pulmonary and extrapulmonary in immigrant TB patients and found that the direct costs for the entire TB illness averaged $€ 353$; the total direct cost to the patients ranged from $€ 0$ to $€ 3961$ [9]. The costs during the pre-diagnostic period were slightly higher for people with extrapulmonary TB than that with pulmonary TB. Most of the costs were incurred for indirect costs; extrapulmonary TB patients lost more work time during the pre-diagnostic period than pulmonary TB patients.

TB is still a major public health problem in Yemen. Yemen is considered a high-burden country in the Region based on the evaluation of TB conducted in 1990-1991 in the Nationwide Survey of the Tuberculin Testing among school children [10]. The annual expected incidence of new pulmonary TB smear-positive (NSS+) was 8480 , and equal number of other forms of TB was estimated. The most recent estimates of TB in Yemen show that the annual incidence of NSS+ cases is 37 per 100000 population (7297 NSS+ cases per year) and the annual incidence for other forms of TB is 45 per 100000 population (8874 cases per year). Sana'a, the capital city of Yemen, has the highest number of extrapulmonary $\mathrm{TB}$ cases and has the second highest number of cases of all forms of TB compared to other provinces [11].

The aim of this study was to analyse the economic burden of pulmonary and extrapulmonary TB in Sana'a from both the patient and the health services perspectives. The findings will provide the government with detailed data concerning the cost of $\mathrm{TB}$ diseases per patient and will enable estimates to be made of the public health expenditure on TB treatment.

\section{Methods}

A prospective pharmacoeconomic evaluation was carried out for diagnosis and treatment of pulmonary and extrapulmonary TB.

\section{Study setting and sample}

The study population consisted of all TB patients who started a new course of TB treatment and completed their treatment successfully at Sana'a TB centre and 6 other health facilities from 1 March 2008 to 10 August 102009.

The sample size was calculated using the equation $n=N /\left(1+\mathrm{Ne}^{2}\right)$, where $n=$ sample size; $N=$ population size (1193); $e=$ margin of error (0.05). the sample size was increased by $20 \%$ to account for drop-outs.

The time away from work was counted for the 48 employed patients who came during the period of study. The length of follow-up for cost calculation during the treatment period was 8 months for pulmonary TB patients and 12 months for extrapulmonary TB patients. Patients who transferred from Sana'a TB centre and health facilities to complete their treatment in other health centres in other provinces, and patients who defaulted or died were excluded from the analysis.

\section{Drug regimen}

The drug regimens prescribed were according to the type of TB disease. If diagnosed with susceptible positive pulmonary TB, in the initial (intensive) phase the patient was given: 2HRZS(E), i.e. isoniazid, rifampicin, pyrazinamide and either streptomycin or ethambutol, given once daily for 2 months ( 8 weeks). In the continuation phase, the patient was given: $6 \mathrm{HT}$, i.e. isoniazid and thioacetazone daily for 6 months. For extrapulmonary and negative pulmonary $\mathrm{TB}$ the regimen was 2HTS(E), i.e. isoniazid, thioacetazone and either streptomycin or ethambutol in the intensive phases and $10 \mathrm{HT}$ in the continuation phase, i.e. isoniazid and thioacetazone daily for 10 months. The patients were followed from the first day of treatment until the end of treatment when they came to collect their medication.

\section{Estimation of costs}

The total cost stems from 2 sources: direct and indirect costs. Direct medical costs include the cost of prescribed medication, diagnostic laboratory tests, $\mathrm{X}$-ray films, reagents and supplies for $\mathrm{X}$-ray departments as well as the cost of the time taken by health professional workers. Direct nonmedical costs include the cost of transportation to and from centres for the patient, and telephone, electric and water bills for health facilities. The estimated cost of building, vehicles and furniture were excluded because of the difficulty in estimating these kinds of cost. The indirect cost related to the loss of time from work due to illness was estimated using a specially-designed data collection form, which enquired about patients' employment status; amount of time 
lost if ill, and net monthly salary when healthy. The average cost per patient for time away from work was calculated by multiplying the average number of days away from work due to illness with the average of the patient's monthly income when healthy and working.

All costs of anti-TB drugs were calculated according to the standard government drug price. The staff time was calculated by estimating the time spent for each patient in each section. Then, the net salary of health workers per minute was multiplied by the time per taken (in minutes) for each patient [12].

Costs were obtained from the TB centres and health facilities in Sana'a; all were calculated in Yemen rials (YR) and converted to the United States dollars (US\$) at the 2009 exchange rate of US\$ 1 = YR 200 .

\section{Analysis}

SPSS for Windows, version 12.0 was used for data analysis.

\section{Results}

\section{Patient characteristics}

A total of 390 patients were included. Of these, 15 patients transferred from Sana' a TB centre and health facilities to complete their treatment in other health centres in other provinces, $24 \mathrm{pa}$ tients defaulted and 7 patients died and they were excluded from the analysis. In the end 160 pulmonary TB patients and 160 extrapulmonary TB patients were included in the analysis. For pulmonary TB $46 \%$ were male, while for extrapulmonary TB 38\% male. The majority of pulmonary and extrapulmonary $\mathrm{TB}$ patients ranged in age from 15 to 54 years ( $89 \%$ and $93 \%$ respectively).

\section{Total costs}

The total cost, both direct and indirect, for TB treatment for the 160 pulmonary TB patients was US\$ 22 778, i.e. US\$ 142.4 per patient; $76 \%$ of this cost was borne by the patients themselves and $23.9 \%$ by the health services (Table 1). The average cost to the patient was US\$ 108.4 compared with US\$ 34.0 for the health services per patient. For extrapulmonary TB treatment, the total cost was US\$ 58 682.2, i.e. US\$ 366.76 per patient; $89.4 \%$ of this cost was borne by the patients. The average cost to the patient was US\$ 328.0 compared with US\$ 38.8 for the health services per patient.

Tables 2 and 3 show the break-down of costs for diagnosis and treatment of pulmonary and extrapulmonary TB patients for the health services and the patients.

\section{Cost to the health services}

The direct medical costs included medication, X-ray examination, laboratory costs and health staff costs.

Drugs were distributed free of charge to the patients. The total costs were US\$ 3225.6 for the pulmonary TB patients (US\$20.2 per patient) and US\$ 4838.4 for the extrapulmonary TB patients (US\$ 30.2 per patient) (Table 2).

A total of 320 X-ray films were used for pulmonary $\mathrm{TB}$ patients at a cost of
US\$ 0.80 per film, giving a total cost of US\$256; adding supplies and reagents brought the total cost to US\$ 416.0, an average cost of US\$2.6 for each patient cured following completion of treatment. Of these costs, each patient paid US\$ 1 for the first X-ray while any later X-rays were free. Bacteriological examination of sputum is the only way to confirm the diagnosis of pulmonary TB in most developing countries. Sana'a TB Centre only does acid fast bacilli (AFB) for positive pulmonary TB. Patients are required to pay US\$ 1 for the first visit and nothing for any other tests. The cost of an AFB test preparation for each patient was US\$2.2.

The total cost for the public health staff was US\$ 833.8 in the case of pulmonary TB and US\$ 395.8 in the case of extrapulmonary TB (Table 2). The estimated overheads cost per patient was US\$ 0.70 for pulmonary TB cases and US\$ 1.20 for extrapulmonary TB cases.

Government expenditure on drug treatment for the pulmonary TB cases was slightly more than half of the total costs to the health services (59.3\%). Health staff time was the next highest cost (15.3\% of total expenditure); the $\mathrm{X}$-ray examination cost represented $7.6 \%$ of the total (Table 3 ). For extrapulmonary TB patients, government expenditure on drugs was $77.9 \%$ of the total expenditure, followed by stationery costs $(12.6 \%)$.

\section{Cost to the patients}

Medical costs for patients included physician costs and laboratory and

\begin{tabular}{|c|c|c|c|c|c|c|}
\hline \multirow[t]{2}{*}{ Total cost to: } & \multicolumn{3}{|c|}{ Pulmonary TB $(n=160)$} & \multicolumn{3}{|c|}{ Extrapulmonary TB $(n=160)$} \\
\hline & Total cost (US\$) & $\begin{array}{c}\text { Cost per } \\
\text { patient (US\$) }\end{array}$ & $\%$ of total cost & Cost (US\$) & $\begin{array}{c}\text { Cost per } \\
\text { patient (US\$) }\end{array}$ & $\%$ of total cost \\
\hline Patients & 17336.0 & 108.4 & 76.1 & 52472.0 & 328.0 & 89.4 \\
\hline Public health services & 5442.7 & 34.0 & 23.9 & 6210.2 & 38.8 & 10.6 \\
\hline Total & 22778.7 & 142.4 & & 58682.2 & 366.8 & \\
\hline
\end{tabular}




\begin{tabular}{|c|c|c|c|c|c|c|}
\hline \multirow[t]{3}{*}{ Item } & \multicolumn{3}{|c|}{ Pulmonary TB $(n=160)$} & \multicolumn{3}{|c|}{ Extrapulmonary TB $(n=160)$} \\
\hline & $\begin{array}{l}\text { Total cost to the } \\
\text { public health } \\
\text { services }\end{array}$ & $\begin{array}{c}\text { Cost per } \\
\text { patient }\end{array}$ & $\%$ & $\begin{array}{l}\text { Total cost to the } \\
\text { public health } \\
\text { services }\end{array}$ & $\begin{array}{l}\text { Cost per } \\
\text { patient }\end{array}$ & $\%$ \\
\hline & US\$ & US\$ & & US\$ & US\$ & \\
\hline Drugs & 3225.6 & 20.2 & 59.3 & 4838.4 & 30.2 & 77.9 \\
\hline $\mathrm{X}$-ray examination & 416.0 & 2.6 & 7.6 & - & - & - \\
\hline Laboratory tests & 336.9 & 2.2 & 6.2 & - & - & - \\
\hline Health staff time & 833.8 & 5.2 & 15.3 & 395.8 & 2.5 & 6.4 \\
\hline Stationery & 523.0 & 3.3 & 9.6 & 785.0 & 4.9 & 12.6 \\
\hline Overheads & 107.4 & 0.7 & 2.0 & 191.0 & 1.2 & 3.1 \\
\hline Total cost & 5442.7 & 34.0 & & 6210.2 & 38.8 & \\
\hline
\end{tabular}

X-ray costs. For both pulmonary and extrapulmonary $\mathrm{TB}$ the cost per patient for physician fees was US\$ 0.25 . However for laboratory tests/X-rays, the cost per patient was US $\$ 2.00$ for pulmonary TB and US\$ 182.0 for extrapulmonary TB.

The transportation cost per patient was US\$ 33 for pulmonary TB patients, and US\$ 94 for extrapulmonary TB patients. The cost of time away from work for the 48 patients (27 pulmonary and 21 extrapulmonary) who were working was calculated. The total working time lost was 622 days and the average time spent away from work was 12.9 days per patient, for pulmonary and extrapulmonary together. The loss in terms of money depended on the patients' monthly income: the average monthly income for pulmonary TB cases was US\$ 170.20 and US\$ 120.70 for extrapulmonary TB patients. The average cost to the patient for time away from work was US $\$ 73.2$ and US\$ 51.9 for pulmonary and extrapulmonary TB patients respectively.

The mean cost to patients for pulmonary TB treatment, which includes physician fees, laboratory tests, X-rays, transportation and the cost of being away from work, was US\$ 108.4. The greatest cost proportionately to patients for pulmonary TB treatment was time away from work $(67.5 \%$ of the total cost), followed by transportation (30.5\%). For extrapulmonary TB the mean cost to the patients for treatment was US\$ 328.0. The greatest cost proportionately was laboratory and X-ray costs (55.5\%), followed by transportation (28.6\%) and time away from work (15.8\%).

\section{Discussion}

In the present study, the findings are based on actual expenditures paid for TB treatment. Our study shows that the average cost of TB treatment per patient was US\$ 142.40 and US\$366.80 for pulmonary TB and extrapulmonary TB patients respectively. The higher cost for extrapulmonary TB patients is due to the longer period of treatment which needs more expenditure on transportation, in addition to the high cost of the tests, which are done outside the centres.

Comparing the total cost of treatment of pulmonary TB to other studies, the cost of treatment of pulmonary $\mathrm{TB}$ in our study was very much lower than that estimated by WHO in 2000 in developed countries (US\$ 276-1546) [13].The cost in Yemen was also lower

\begin{tabular}{|c|c|c|c|c|c|c|}
\hline \multirow[t]{3}{*}{ Item } & \multicolumn{3}{|c|}{ Pulmonary TB $(n=160)$} & \multicolumn{3}{|c|}{ Extrapulmonary TB $(n=160)$} \\
\hline & Total cost & Cost per patient & $\%$ & Total cost & Cost per patient & $\%$ \\
\hline & US\$ & US\$ & & US\$ & US\$ & \\
\hline Physician fees & 40.0 & 0.25 & 0.2 & 40.0 & 0.25 & 0.1 \\
\hline Laboratory \& X-ray fees & 320.0 & 2.00 & 1.8 & 29120.0 & 182.00 & 55.5 \\
\hline Transportation & 5280.0 & 33.00 & 30.5 & 15008.0 & 93.80 & 28.6 \\
\hline Time away from work & 11696.0 & 73.10 & 67.5 & 8304.0 & 51.90 & 15.8 \\
\hline Total cost & 17336.0 & 108.40 & & 52472.0 & 328.00 & \\
\hline
\end{tabular}


than that reported in studies done in Malaysia (US\$ 916.40), South Africa (US\$ 890.50), Uganda (US\$ 403.20) and Pakistan US\$ 310.00 [7,14-16]. However, a comparable cost was reported in India, around US\$ 171 [17]. The lower cost of expenditure in Yemen can be attributed to the lower number of diagnostic tests (AFB only for diagnosing pulmonary $\mathrm{TB}$ ) and the absence of hospitalization.

The costs to the health services in the present study were US\$ 34.0 and US\$ 38.8 per patient for pulmonary $\mathrm{TB}$ and extrapulmonary $\mathrm{TB}$ respectively. The cost of health services (for public TB centre) was slightly lower in pulmonary $\mathrm{TB}$ than in extrapulmonary TB because the extrapulmonary TB patients did not carry out laboratory tests and X-ray examination in the TB centres, as patients went to the centre after having done their diagnostic tests The total direct expenditure to the health services for pulmonary TB treatment in the current study is comparable to the cost reported in a study in India which were US\$30 to US\$43 according to the category of treatment [8]. On the other hand, very high expenditure was found in the study conducted in Haiti where the total direct expenditure for pulmonary TB was US\$ 432 per TB patient [18], while in another study conducted in Malaysia, the total direct expenditure to the health services for pulmonary TB was US\$ 189.50 [7].

The largest proportion of direct medical costs for pulmonary TB was drug cost (59.3\%). In the case of extrapulmonary TB, the TB medication constituted $77.9 \%$ of the total cost of the illness. The proportionate cost of drug treatment was higher in extrapulmonary $\mathrm{TB}$ than in pulmonary $\mathrm{TB}$ because extrapulmonary $\mathrm{TB}$ requires longer treatment and more expensive drugs. Lower proportionate costs for pulmonary TB drugs of the total costs of illness were reported in other studies: $32.4 \%, 25 \%$ and $37.8 \%$ respectively $[7,8,12]$. Similar findings were reported in a study in Uganda although there were additional costs associated with streptomycin (water for injection and syringe) [15].

Overall, the percentage cost to the patients in the present study was much higher than to the health services, $76.1 \%$ and $89.4 \%$ for pulmonary TB and extrapulmonary TB respectively. Comparable results for pulmonary TB were reported in studies conducted in Uganda [19], Malaysia [7] and Tanzania [5] where the proportionate cost to the patients was $70.0 \%, 79.5 \%$ and $84.2 \%$ respectively. These costs are high as the pulmonary TB patients have to visit the centres either weekly (in the initial phase) or monthly (in the continuous phase) while extrapulmonary TB patients have to visit it monthly to get their medicines.

The cost to the patient associated with the loss of productivity for pulmonary TB was US\$ 73.1, while for extrapulmonary TB patient it was US\$ 51.9. The lower cost to extrapulmonary TB patients could be due to the high fever associated of pulmonary TB patients, which could have resulted in more days lost from work and higher loss of productivity.

\section{Study limitations}

We included only patients living in urban areas and with access to provincial facilities. This limits the generalizability of the results and the estimated costs may be more than the actual costs for rural residents. The study did not quantify the intangible costs (increased pain and disability, affecting quality of life), nor extra expenditure of the patient for, for example, having a companion accompany them during visits to the centres for treatment. Thus the cost to patients may be higher than found here.

\section{Conclusions}

The study found that the cost of extrapulmonary $\mathrm{TB}$ is higher compared to pulmonary TB especially for the patient, which reflects the delayed diagnosis and longer duration of treatment. $\mathrm{Pa}$ tients bore the majority of the cost of diagnosis and treatment of pulmonary and extrapulmonary TB. The cost of anti-TB drugs constituted the highest proportion of the costs to the public health services for both pulmonary and extrapulmonary TB. The results of this study may be used by policy-makers, potential donors and health service workers to enhance TB diagnosis and treatment services.

\section{Acknowledgements}

This study was funded by Al-Saeed Foundation for Science and Culture, Yemen. We thank all the staff of the TB centre and health facilities in Sana'a Yemen.

\section{References}

1. FitzGerald JM, Houston S. Tuberculosis 8. The disease in association with HIV infection. Canadian Medical Association Journal,1999, 161(1): 47-51.

2. WHO Global TB Programme. Anti-tuberculosis drug resistance in the world. Geneva, World Health Organization, 1997.

3. Global tuberculosis control: surveillance, planning and financing Geneva, World Health Organization, 2006.
4. Global tuberculosis control: surveillance, planning, financing. Geneva, World Health Organization, 2008 (WHO/HTM/ TB/2008.393).

5. Wyss K, Kilima P, Lorenz N. Cost of tuberculosis for household and health care providers in Dar es Salaam, Tanzania. Tropical Medicine \& International Health, 2001, 6:60-68.

6. The economic impacts of tuberculosis. Geneva, World Health Organization, 2000. 
7. Elamin El et al. Cost of illness of tuberculosis in Penang, Malaysia. Pharmacy World \& Science, 2008, 30:281-286.

8. Muniyandi M, Rajeswari R, Rani B. Costs to patients with tuberculosis treated under dots programme. Indian Journal of Tuberculosis, 2005, 52:188-196.

9. Kik SV et al. Direct and indirect costs of tuberculosis among immigrant patients in the Netherlands. BMC Public Health, 2009, 9:283

10. Al-Akhali A et al Nationwide survey on the prevalence of antituberculosis drug resistance in the Republic of Yemen, 2004. International Journal of Tuberculosis and Lung Disease, 2007, 11(12):1328-1333.

11. Al-Absi, A. N. Annual report of the national tuberculosis control program. Republic of Yemen, Ministry of public health \& population (2007)

12. Islam $\mathrm{M}$ et al. Cost effectiveness of community health workers in tuberculosis control in Bangladesh. Bulletin of the World Health Organization, 2002, 80:445-450.

13. Ahlburg DA. Theeconomic impact oftuberculosis. Geneva, World Health Organization, 2000 (Stop TB Initiative series) (http:// whqlibdoc.who.int/hq/2000/WHO_CDS_STB_2000.5.pdf, accessed 8 March 2012).
14. Floyd K, Wilkinson D, Gilks C. Comparison of cost effectiveness of directly observed treatment (DOT) and conventional delivered treatment for tuberculosis: experience from rural South Africa. BMJ (Clinical Research Ed.), 1997, 315:1407-1411.

15. Sanderson PR. An economic evaluation of alternative programme designs for tuberculosis control in rural Uganda. Social Science \& Medicine, 1995, 40:1203-1212.

16. Khan MA et al. Costs and Cost effectiveness of different DOT strategies for the treatment of tuberculosis in Pakistan cost. Health Policy and Planning, 2002, 17:178-186.

17. Rajeswari R, Balasubramanian R, Muniyandi M. Socioeconomic impact of tuberculosis on patient and family in India. International Journal of Tuberculosis and Lung Disease, 1999, 3:869-877.

18. Jacquet $\mathrm{V}$ et al. Impact of DOTS expansion on tuberculosis related outcomes and costs in Haiti. BMC Public Health, 2006, 6:209.

19. Guwatudde D et al. Burden of tuberculosis in Kamala, Uganda. Bulletin of the World Health Organization, 2003, 81:799-805.

\section{Priorities in operational research to improve tuberculosis care and control}

In 2010, the Stop TB Partnership, the World Health Organization Stop TB Department and the Global Fund to Fight AIDS, Tuberculosis and Malaria jointly organized an expert meeting and workshop on operational research, followed by international consultations. The goal was to identify priority areas in which knowledge gaps hamper optimal implementation of TB control activities. The outcome of these activities formed the basis of Priorities in operational research to improve tuberculosis care and control. This book is very timely for building the evidence base for effective implementation of TB programmes. It provides a clear road map of priorities in operational research to help countries improve implementation of TB control activities in critical areas. The priority operational research questions outlined in this report are also aligned with the Stop TB Partnership's Global Plan to Stop TB 2011-2015.

Further information about this and other WHO publications is available at: http://www.who.int/publications/en/ 